Magnetization and Electrical Resistivity of Terbium Single Crystals

D. E. HEGLAND, S. LEGVOLD, AND F. H. SPEDDING

Institute for Atomic Research and Department of Physics

Iowa State University, Ames, Iowa

\title{
ABSTRAC T
}

Magnetic moment measurements have been made on single crystals of terbium (hcp) with fields of 50 to 18,000 oe applied along the $\langle 11 \overline{2} 0\rangle(\mathrm{a}$-axis), $<10 \mathrm{~T} 0\rangle$ (b-axis), and $<0001\rangle$ (c-axis) directions. The temperature range covered was 1.4 to $500^{\circ} \mathrm{K}$. The b-axis was found to be the easy direction of magnetization with an extrapolated saturation moment of 9.34 Bohr magnetons per atom. Anisotropy was observed in the basal plane below the ferromagnetic Curie temperature but not above. A weak antiferromagnetic state was observed between $229^{\circ} \mathrm{K}$ and the ferromagnetic Curie point at $221^{\circ} \mathrm{K}$. Electrical resistivity measurements were made from 4.2 to $360^{\circ} \mathrm{K}$ (a-axis crystal) and from 4.2 to $300^{\circ} \mathrm{K}$ (b and c-axis crystals). The basal plane curve changed slope at 221 and $229^{\circ} \mathrm{K}$. The c-axis curve changed slope at $221^{\circ} \mathrm{K}$, exhibited a nearly symmetrical maximum about $226^{\circ} \mathrm{K}$, and returned to linear behavior near room temperature. The application of a magnetic field of 11 koe along a b-axis caused the pronounced maximum at $226^{\circ} \mathrm{K}$ to disappear.

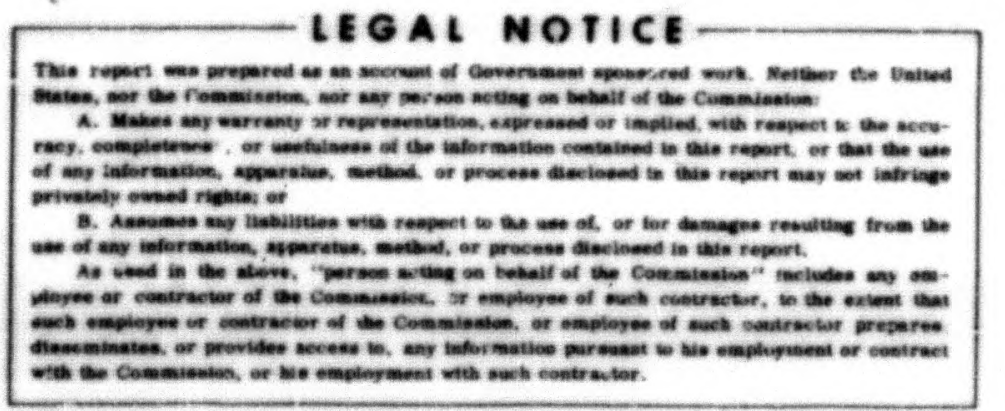

Facsimile Prices s_e.
Microfilm Price s
Avoiloble from the
Office of Technical Services
Depertment of Commerce
Woshington 25, D. C.




\section{INTRODUCTION}

Thoburn et al. ${ }^{1}$ examined polycrystalline terbium magnetically and observed Curie-Weiss behavior above $250^{\circ} \mathrm{K}$ with a paramagnetic Curie temperature of $232 \pm 2^{\circ} \mathrm{K}$. Weak antiferromagnetic ordering was observed between approximately $218^{\circ} \mathrm{K}$ and the Néel point at $230^{\circ} \mathrm{K}$. An applied field of 800 oe was found to be sufficient to quench the antiferromagnetic state. Below $80^{\circ} \mathrm{K}$ the magnetic moment was found to be nearly temperature independent, in agreement with the earlier work of Leipfinger. ${ }^{2}$

Arajs and Colvin ${ }^{3}$ found that polycrystalline terbium follows a Curie-Weiss law up to $1500^{\circ} \mathrm{K}$, and they report an experimental paramagnetic Curie temperature of $236^{\circ} \mathrm{K}$ and an intrinsic magnetic moment of 9.62 Bohr magnetons.

The heat capacity of terbium has been measured by Jennings et al. ${ }^{4}$ over the range 15 to $350^{\circ} \mathrm{K}$. They observed two anomalies, a "bump" at about $221^{\circ} \mathrm{K}$ and a sharp peak at $227.7^{\circ} \mathrm{K}$.

Neutron diffraction studies of a terbium single crystal by Koehler et al. ${ }^{5}$ have revealed that terbium has a helical magnetic structure in the antiferromagnetic region with the magnetic moments parallel to the basal plane and the c-axis as the screw axis. They found a Néel temperature of $230 \pm 1^{\circ} \mathrm{K}$ and observed the onset of ferromagnetism at $223 \pm 1^{\circ} \mathrm{K}$.

The electrical resistivity of polycrystalline terbium has been measured by Colvin et al. ${ }^{6}$ who observed a sharp change in slope at $229^{\circ} \mathrm{K}$ and a slight increase in slope with increasing temperature at $219^{\circ} \mathrm{K}$. 


\section{EXPERIMENTAL PROCEDURE}

The single crystals of terbium used in this investigation were grown from a piece of polycrystalline terbium metal which was prepared by methods previously reported. ${ }^{7,8}$ A strain-anneal techn-que described by Hall et al. ${ }^{9}$ and modified by H. E. Nigh of this Laberatory was used to grow the crystals.

Spectrographic and vacuum fusion analyses of pieces of the crystals from which the samples were cut show the following impurities: c-axis sample:

$\mathrm{Dy} \leq 0.02 \% ; \mathrm{Y}<0.025 \% ; \mathrm{Gd} \leq 0.01 \% ; \mathrm{Sm} \leq 0.075 \% ; \mathrm{Fe}, \mathrm{Ni}, \mathrm{Ca}$, $\mathrm{Cu}, \mathrm{Pr}, \mathrm{Si}$, and Ta present as trace impurities; $\mathrm{O}_{2} \simeq 0.09 \% ; \mathrm{N}_{2} \simeq 0.02 \%$; $\mathrm{H}_{2} \simeq 0.01 \%$.

a- and b-axis samples:

Dy $\leq 0.01 \% ; \mathrm{Y}<0.02 \% ; \mathrm{Gd} \leq 0.01 \% ; \mathrm{Sm} \leq 0.03 \% ; \mathrm{Al}, \mathrm{Ca}, \mathrm{Cu}, \mathrm{Fe}$, $\mathrm{Mg}, \mathrm{Ni}, \mathrm{Si}, \mathrm{Ta}$, and $\mathrm{W}$ present as trace impurities; $\mathrm{O}_{2} \simeq 0.19 \%$; $\mathrm{N}_{2} \simeq 0.02 \% ; \mathrm{H}_{2} \simeq 0.01 \%$.

The experimental procedure and the cryogenic apparatus used for the magnetic measurements in the range 1.4 to $360^{\circ} \mathrm{K}$ have been described by Strandburg et al. ${ }^{10}$ An identical procedure was used in the 300 to $500^{\circ} \mathrm{K}$ range with the dewar replaced by a standpipe furnace.

The experimental procedure and apparatus used for the electrical resistivity measurements have been described by Colvin et al. ${ }^{6}$ EXPERIMENTAL RESULTS

The data for the a-axis crystal are plotted as isotherms in Fig. 1 and as isofield curves in Figs. 2 and 3 . Figure 3 shows the field-dependent 


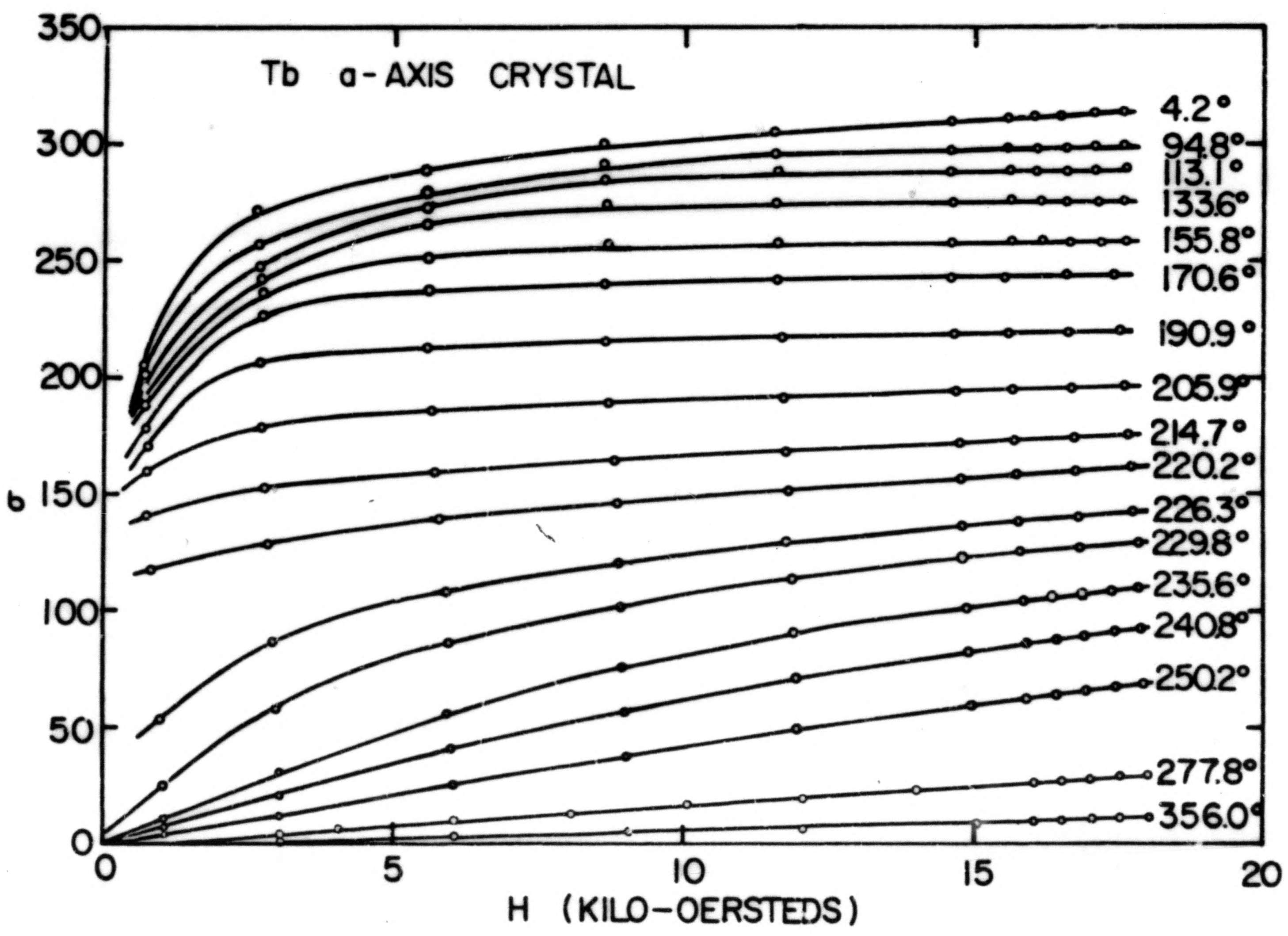

Fig. 1. Magnetic moment/g vs field for the a-axis crystal. 


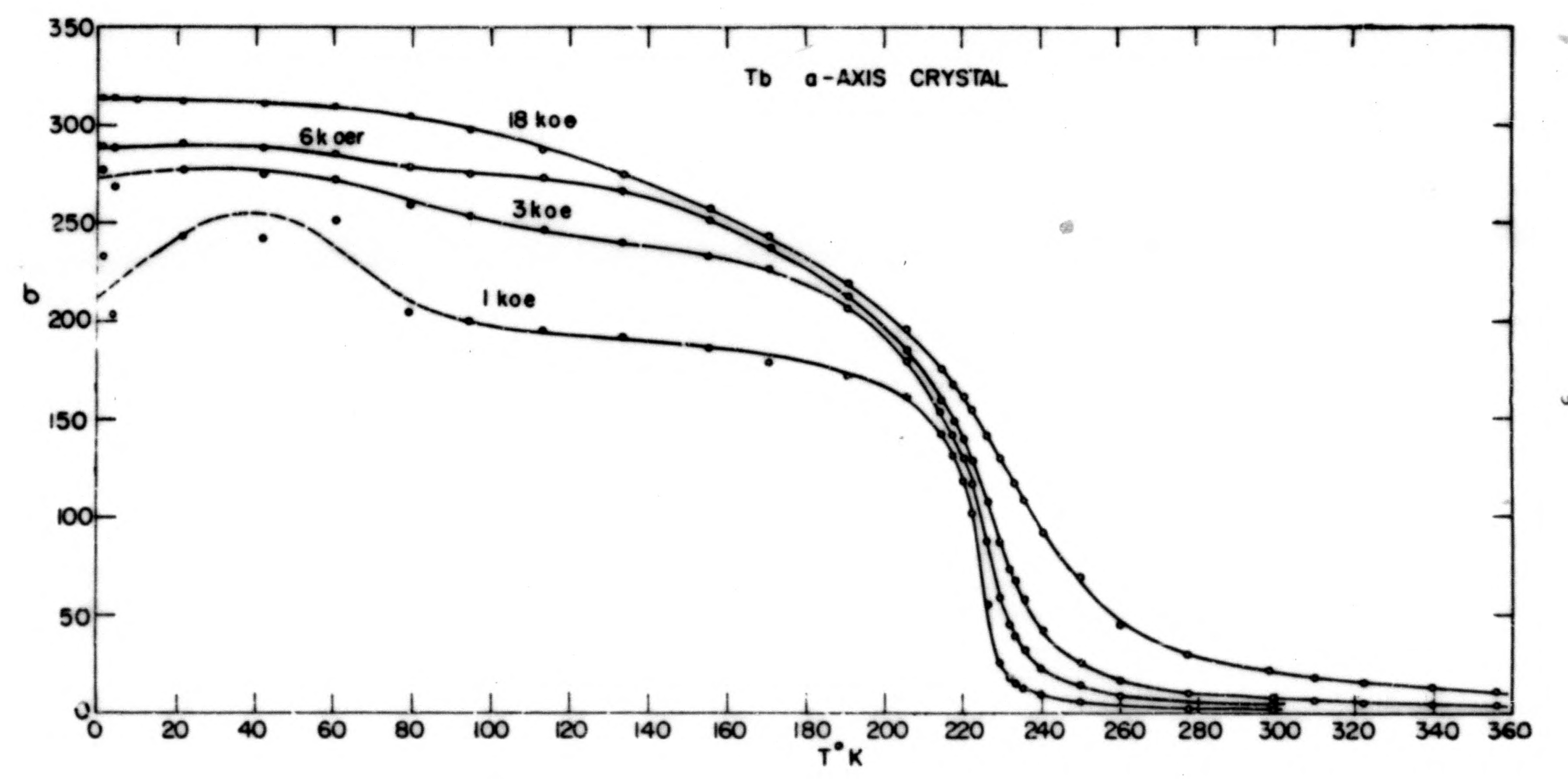

Fig. 2. Magnetic moment/g vs temporature for the a-axis crystal. 


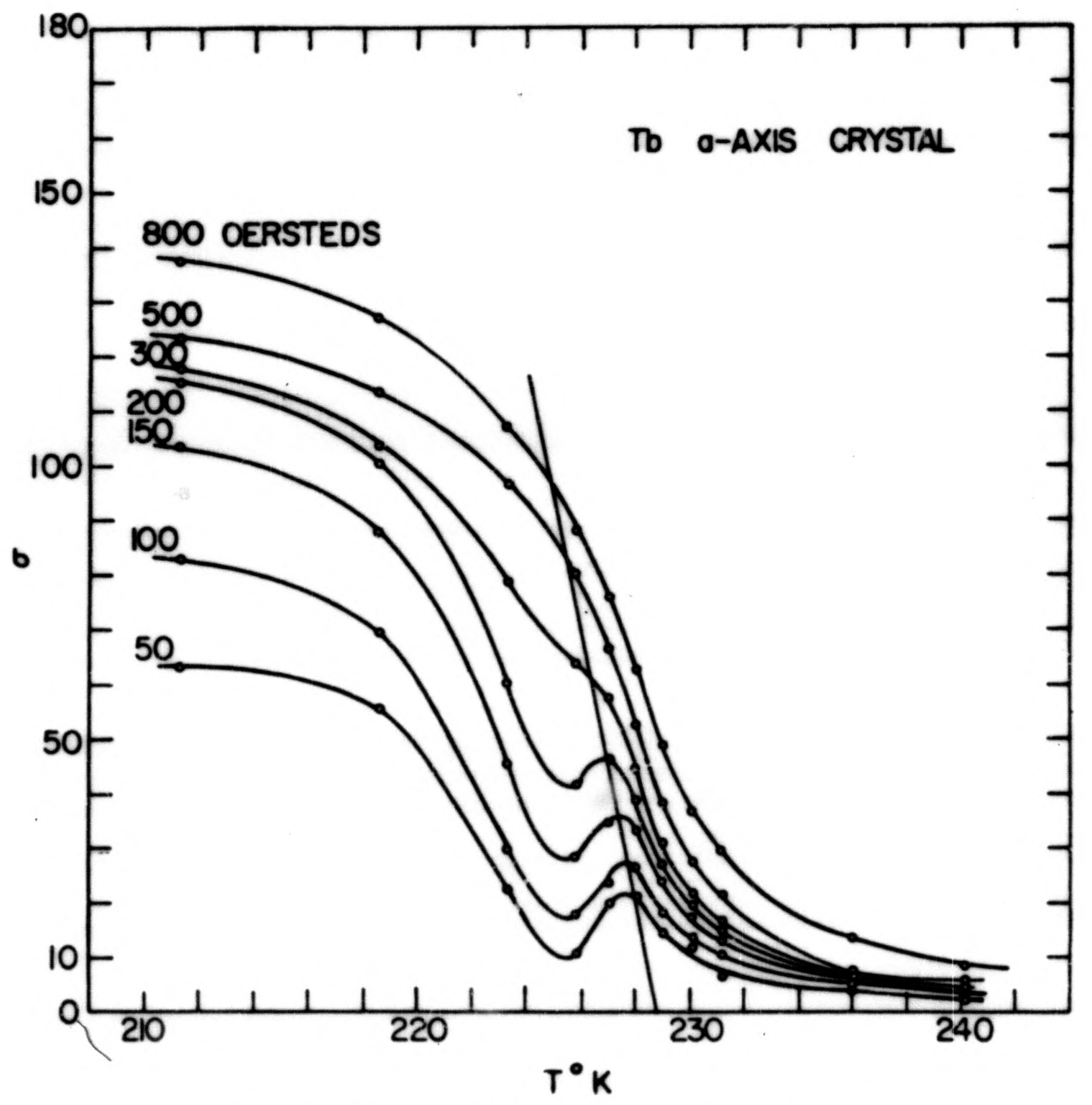

Fig. 3. Magnetic moment/g vs temperature for the a-axis crystal in the neighborhood of the Neel point. 
nature of the Néel point and also the extrapolation to zero field which gives $229 \pm 1^{\bullet} \mathrm{K}$ as the Nél temperature. The paramagnetic data are plotted as the reciprocal of the susceptibility $\left(\frac{1}{X}\right)$ vs $T$ in Fig. 4 . The isotropic magnetic behavior in the basal plane in the paramagnetic region is clearly visible.

The data for the b-axis crystal are plotted as isotherms in Fig. 5 , as isofield curves in Figs. 6 and 7 , and as $\frac{1}{X}$ vs $T$ in Fig. 4. The Néel point in Fig. 7 extrapolated to $229 \pm 1^{\circ} \mathrm{K}$.

The isofield curves are obtained from the isotherms. Since the points of the 1 and 3 koe isofield curves are taken from the steep portions of the isotherms, these curves are probably more qualitative than quantitative.

The magnetic moment per gram, $\sigma$, is plotted $\underline{\mathrm{vs}} \frac{\mathrm{l}}{\mathrm{H}}$ in Fig. 8 for the b-axis crystal and the resulting straight lines extrapolated to $\frac{1}{\mathrm{H}}=0$ to obtain $\sigma_{\infty} \mathrm{T}$. The $\sigma_{\infty} \mathrm{T}$ values are plotted vs $\mathrm{T}^{5 / 2}$ in Fig. 9 , and

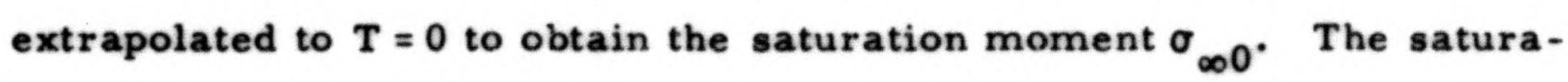
tion moment was $328 \pm 3 \mathrm{emu} / \mathrm{g}$.

Two techniques for determining the ferromagnetic transition temperature were applied to the basal plane data. In one case plots of $\sigma^{2}$ vs $T$ at constant field were extrapolated to $\sigma^{2}=0$ and the resulting T values plotted vs field. This curve was then extrapolated to $H=0$ to yield the ferromagnetic Curie temperature. The result was $221 \pm 2^{\circ} \mathrm{K}$ for both the a- and b-axis crystals. In the other method employed, H was plotted vs $\mathrm{T}$ for several constant values of $\sigma$ and these curves were extrapolated to $\mathrm{H}=0$. The resulting temperatures were plotted vs $\sigma$ and 


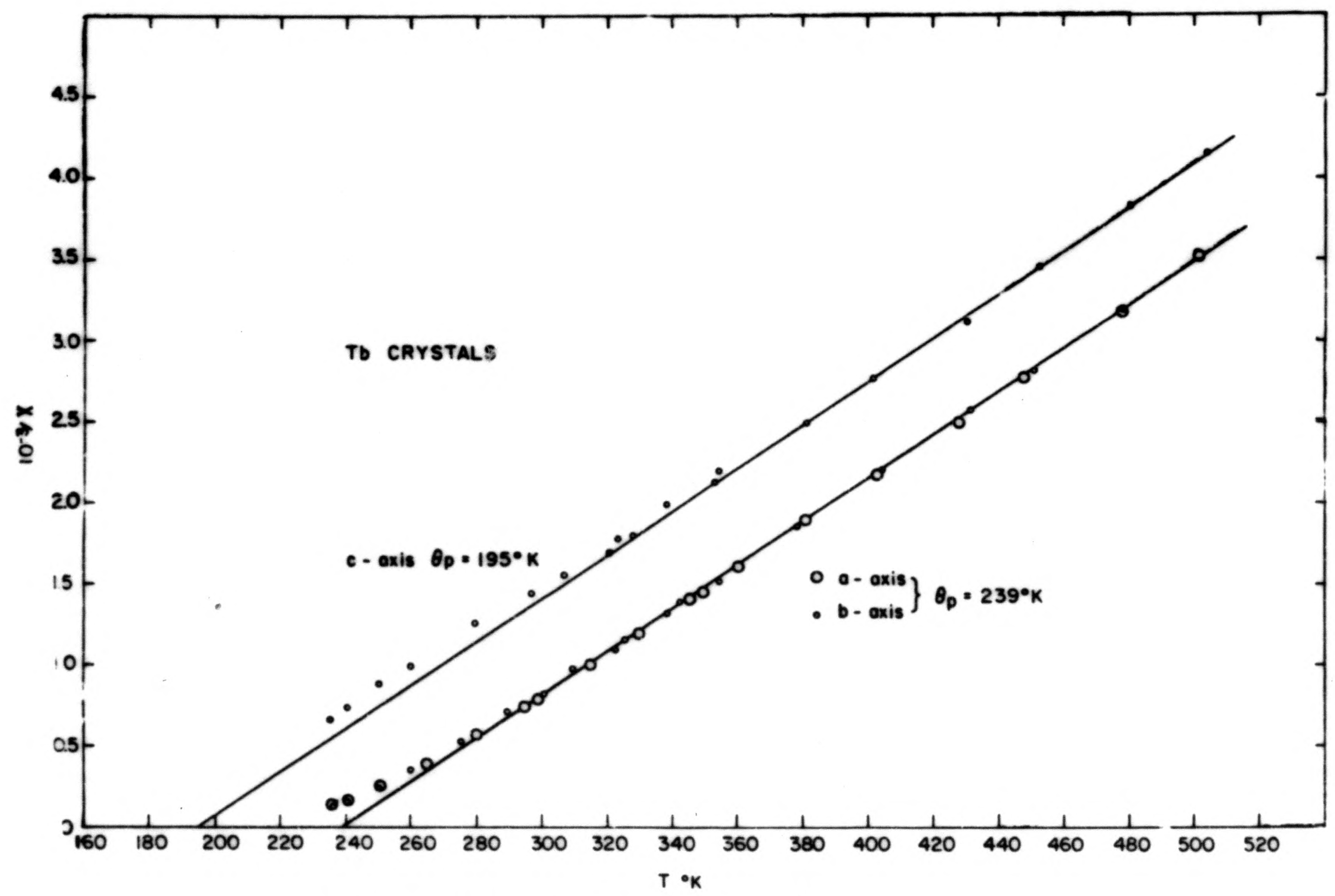

Fig. 4. The reciprocal of the susceptibility $\left(\frac{1}{\mathrm{X}}\right) \mathrm{vs}$ temperature for the a-, b-, and c-axis crystals. 


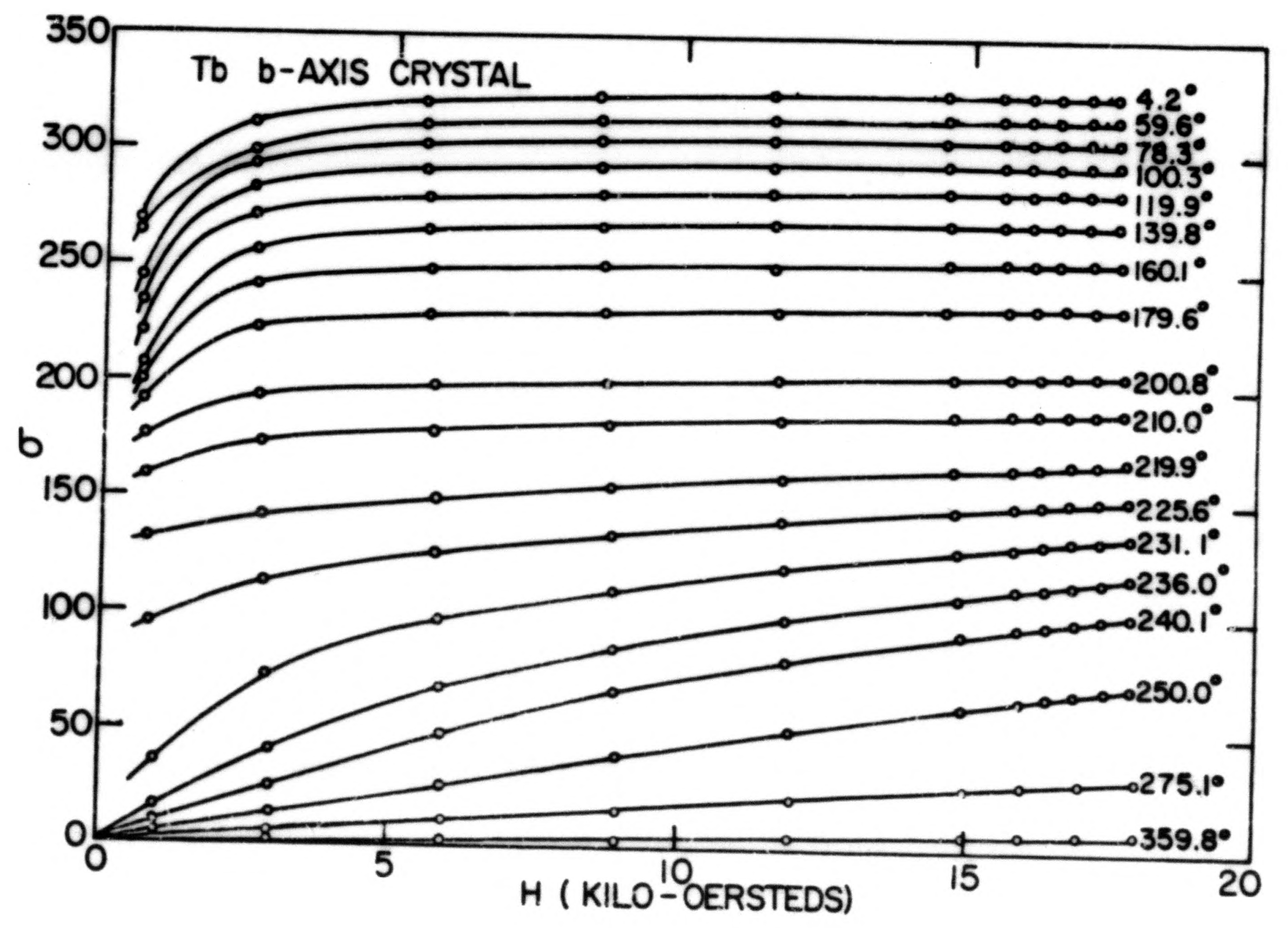

Fig. 5. Magnetic moment/g vs field for the b-axis crystal. 


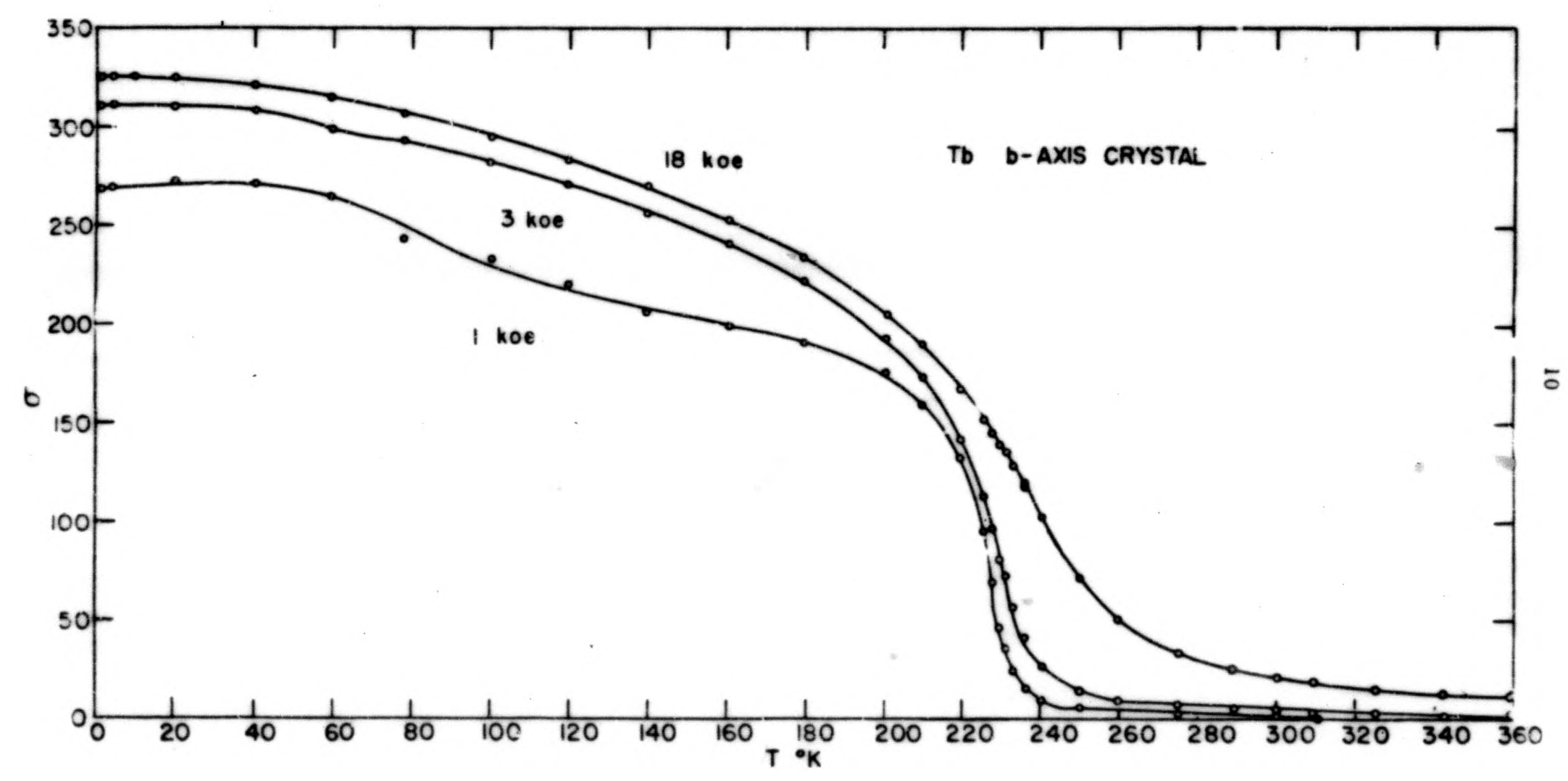

Fig. 6. Magnetic moment/g vs temperature for the b-axis crystal. 


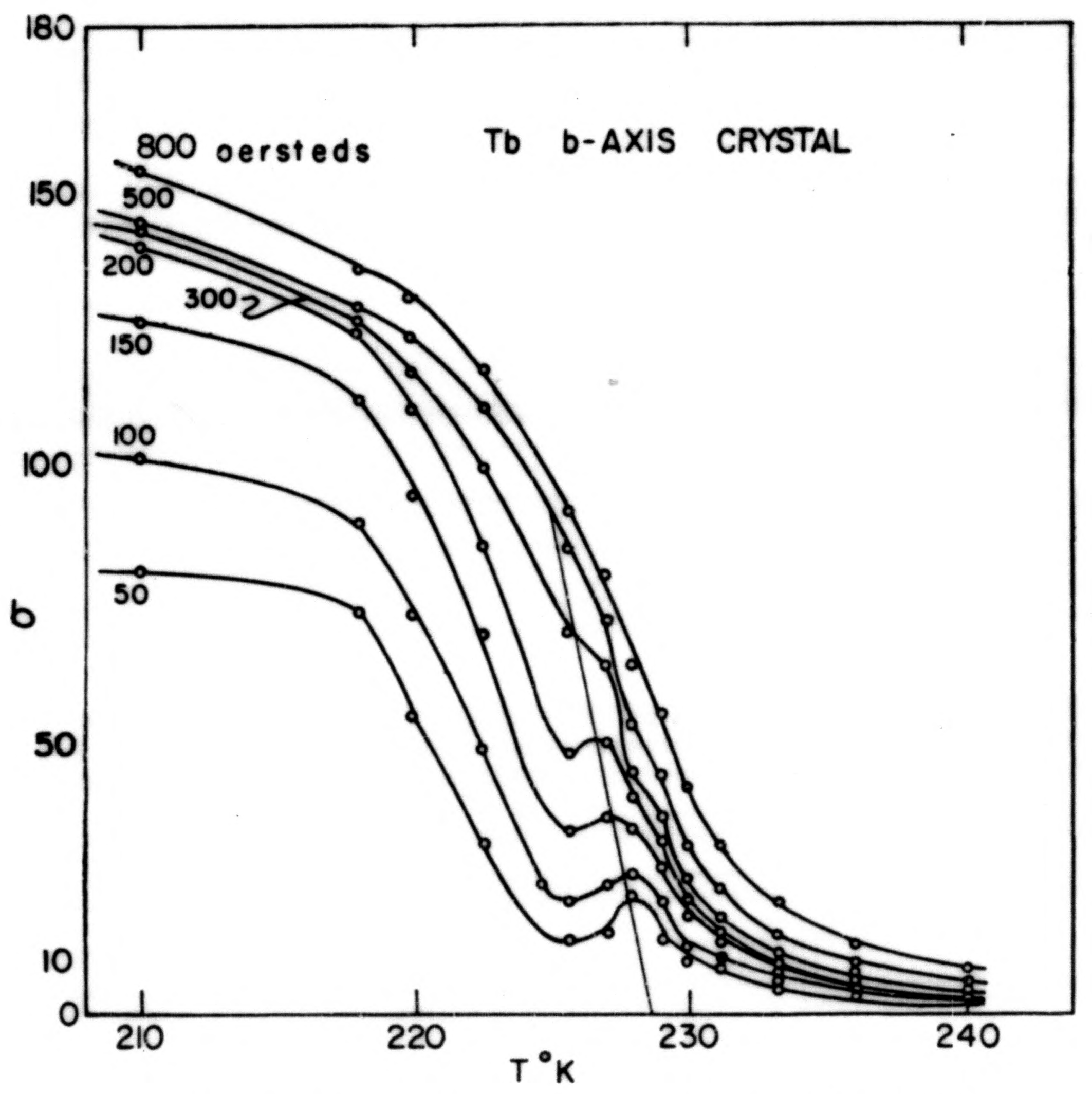

Fig. 7. Magnetic moment/g vs temperature for the b-axis crystal in the neighborhood of the Néel point. 


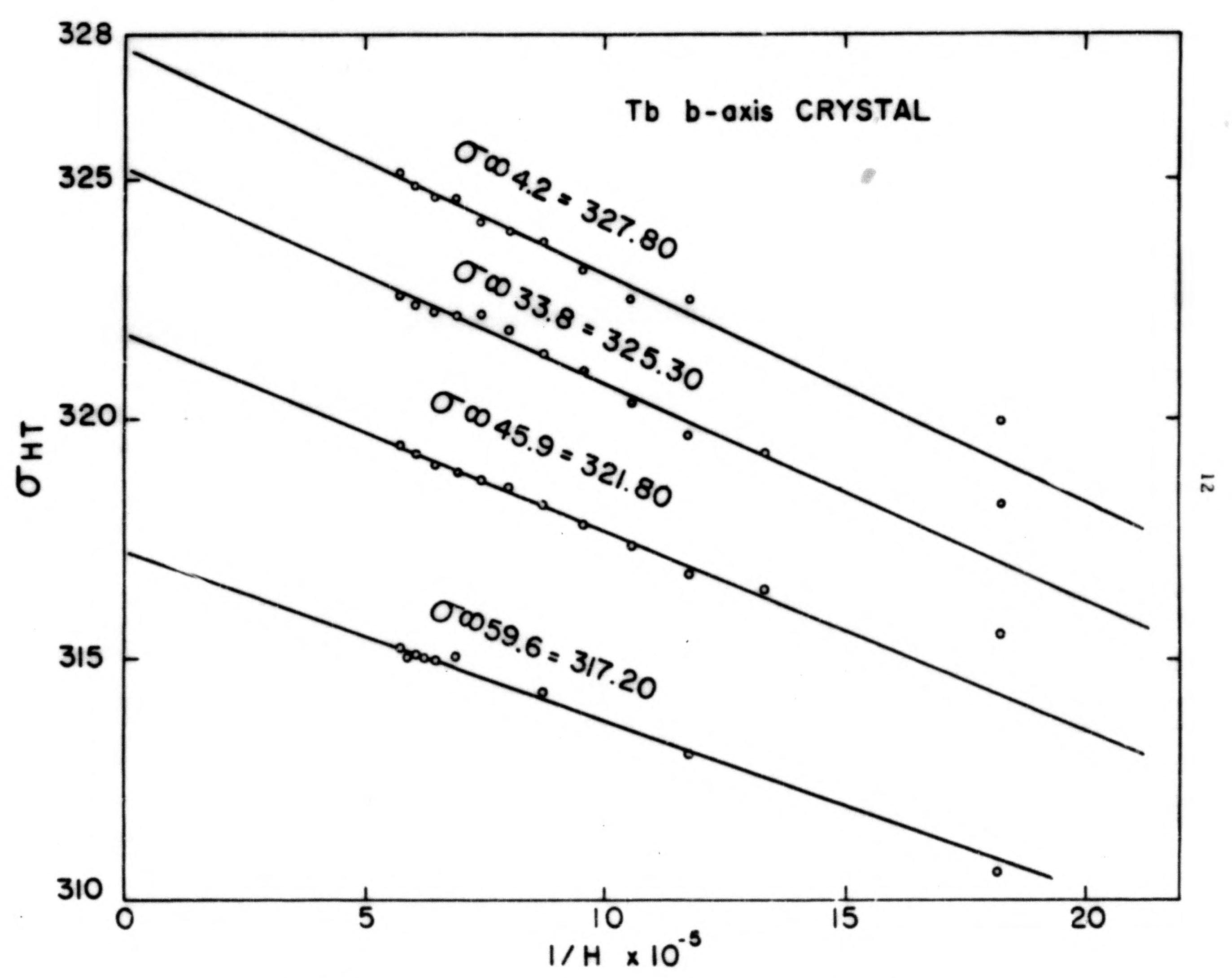

Fig. 8. Magnetic moment/g vs $1 / \mathrm{H}$ for the b-axis crystal. 


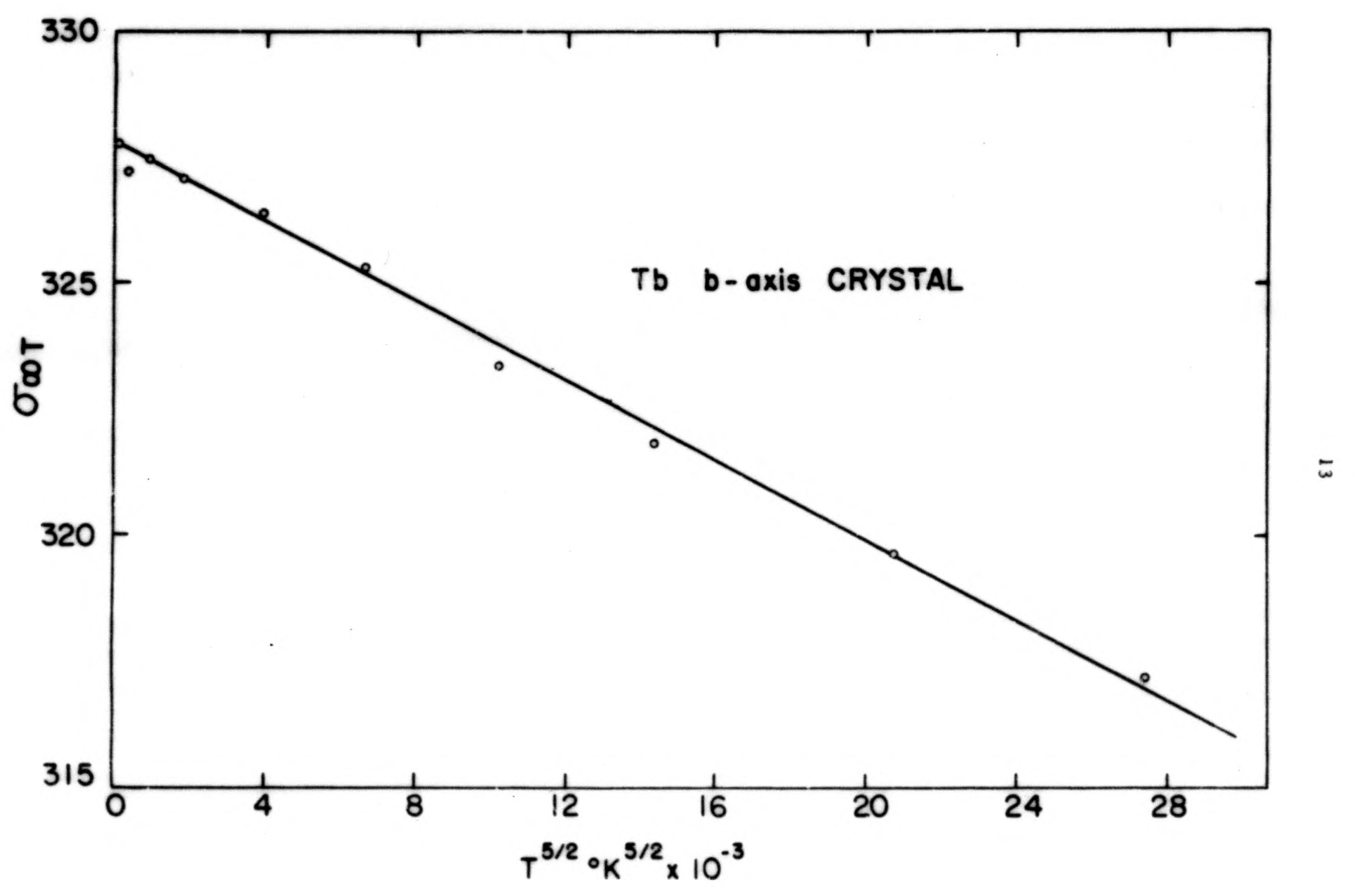

Fig. 9. Saturation ragnetization of the b-axis crystal vs $\mathrm{T}^{5 / 2}$ 
this curve was extrapolated to $\sigma=0$, again yielding the ferromagnetic Curie temperature. The result was $224 \pm 2^{\circ} \mathrm{K}$ for both the a- and b-axis crystals.

The c-axis in terbium is very hard magnetically and as a result the data below $220^{\circ} \mathrm{K}$ is felt to be more qualitative than quantitative in nature. The data at $219.3^{\circ} \mathrm{K}$ and above are plotted as isotherms in Fig. 10. The $219.3^{\circ} \mathrm{K}$ curves are indicative of the general behavior and the lack of reproducibility of the data for temperatures below $220^{\circ} \mathrm{K}$. The c-axis data above $220^{\circ} \mathrm{K}$ are also plotted as $\frac{1}{\mathrm{X}} \underline{\mathrm{vs}} \mathrm{T}$ in Fig. 4 and the anisotropy between the c-axis and the basal plane susceptibilities is clearly evident.

The electrical resistivities (residual subtracted) of the a- and caxis crystals are plotted vs $\mathrm{T}$ in Fig. 11. The residual resistivities were $4.51,5.22$, and $4.13 \mu \mathrm{ohm} \mathrm{cm}$ for the $\mathrm{a}-, \mathrm{b}-$, and c-axis crystals, respectively. Since the a- and b-axis data agreed within the experimental error, only the a-axis data are plotted. Also plotted in Fig. 11 are the experimental data of Colvin et al. ${ }^{6}$ for polycrystalline terbium and a theoretical curve calculated using the relation $\rho_{\text {poly }}=\frac{1}{3}\left(\rho_{c}+2 \rho_{\text {basal plane }}\right)$, which has been verified for yttrium by Alstad et al. 11

A sharp change in slope at $220 \pm 1^{\circ} \mathrm{K}$ is evident in both the c-axis and basal plane curves. The basal plane curve also shows a well-defined change in slope at $229 \pm 1^{\circ} \mathrm{K}$ which does not appear in the c-axis curve. The c-axis curve goes through a nearly symmetrical maximum about $226^{\circ} \mathrm{K}$ and returns to a linear behavior near room temperature. 


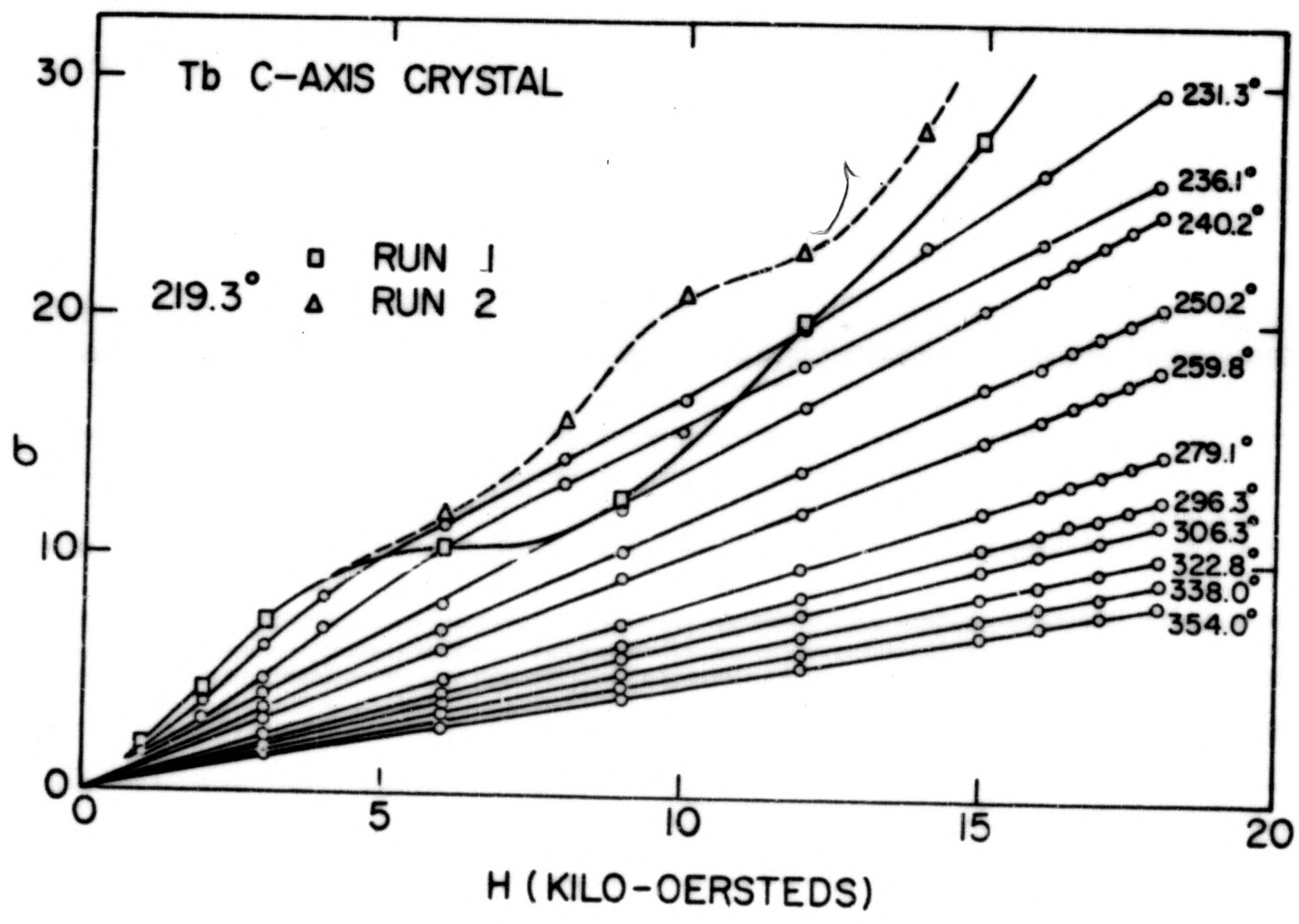

Fig. 10. Magnetic moment/g vs field for the c-axis crystal. 


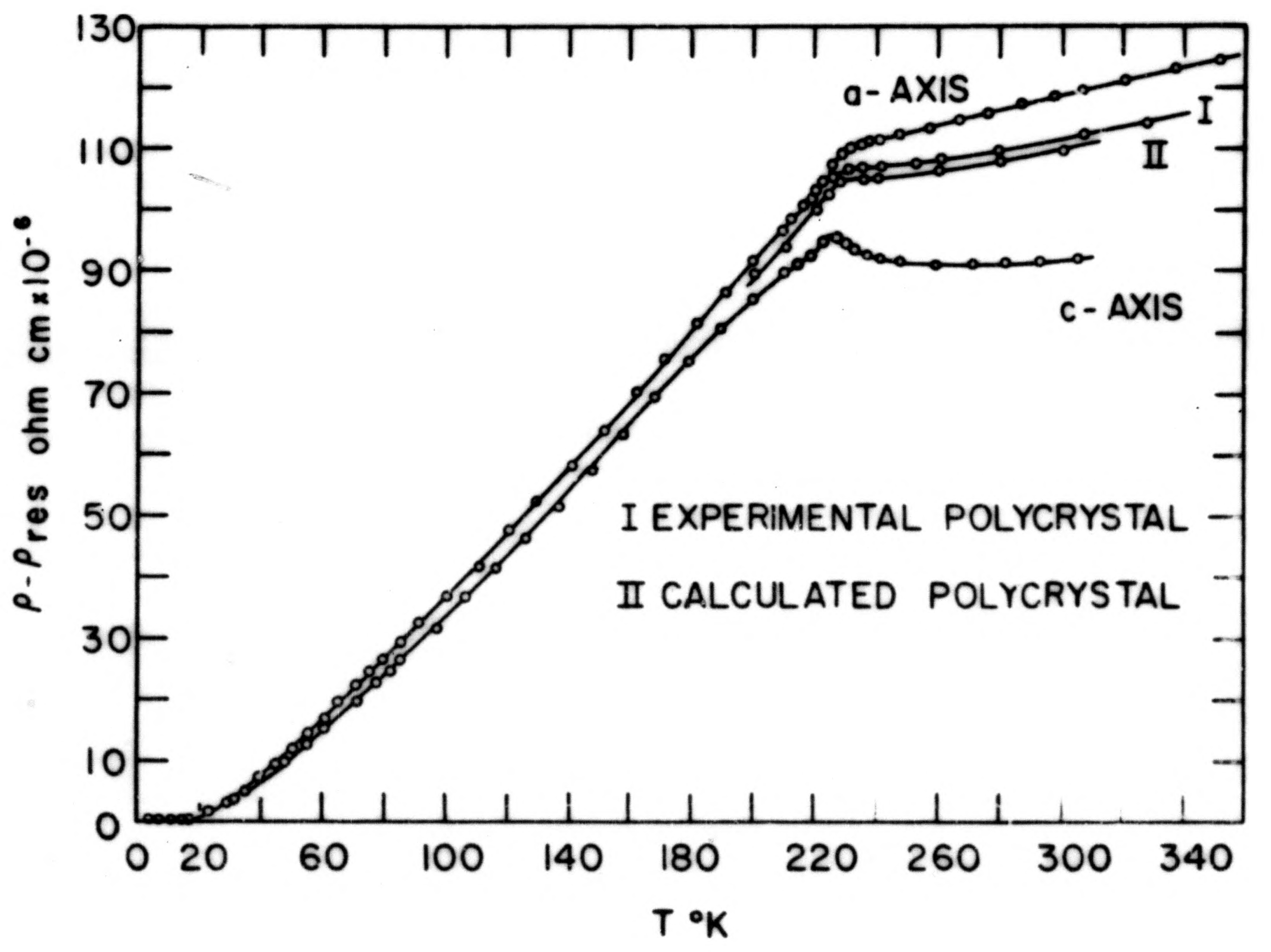

Fig. 11. Electrical resistivity (residual subtracted) vs temperature for the $a-$ and $c$-axis crystals and for a polycrystalline sample. 
The electrical resistivity of the c-axis crystal wi also measured in the temperature range 200 to $250^{\circ} \mathrm{K}$ with magnetic fields applied a ong a b-axis. These data are plotted (residual subtracted) vs $T$ in Fig. 2 . Below $220^{\circ} \mathrm{K}$ the resistivity could be decreased slightiy by the application of the magnetic field, but above $240^{\circ} \mathrm{K}$ the change in resistivity resulting from the application of the field was of the order of magnitude of the experimental error. The peak around $226^{\circ} \mathrm{K}$ could be nearly eliminated by a sufficiently strong magnetic field, leaving a curve very similar to that for gadolinium as reported by Colvin et al. 6

\section{DISC USSION}

The isctherms for the a a cis crystal below $80^{\circ} \mathrm{K}$ showed a small positive slope at high fields. This behavior was a'so observed by Thobu rn et al. ' for polycrystalline terbium. This positive slope indicates that the moments spontaneously align along a b-axis. If this is 30 , then tree spontaneous moment, $\sigma_{6 T}$, for the a-axis snould be equal to $\sigma_{0 T}$ for the b-axis multiplied by $\cos 30^{\circ}$, since the $a-a n d b$-axes lie $30^{\circ}$ apart in the basal plane. When the linear high field parts of the isotherms at iow temperature were extrapolated to $\mathrm{H}=0$ to obtain $\sigma_{0 T}$ it was found that the difference between $\sigma_{0 T}(a-a x i g)$ and $c_{0 T}(b-a x i s)\left(\cos 30^{\circ}\right)$ was less than $3 \%$. Since the experimental error is of the order of $1 \%$ the agreement is regarded as sufficient to substantiate the conclusion that the moments align spontanecusly along a b-axis. Hence the saturation moment, $\sigma_{\infty 0}=328 \pm 3 \mathrm{emu} / \mathrm{g}$, obtained from the $\mathrm{b}$-axis data is the true saturation moment. This value corresponds to $9.34 \pm 0,09$ Bohr magnetons per atom and is to be compared with the theoretical prediction $\mathrm{gJ}=9.0$ Bohr magnetons per atom, calculated assuming $\mathrm{a}^{7} \mathrm{~F}_{6}$ state 


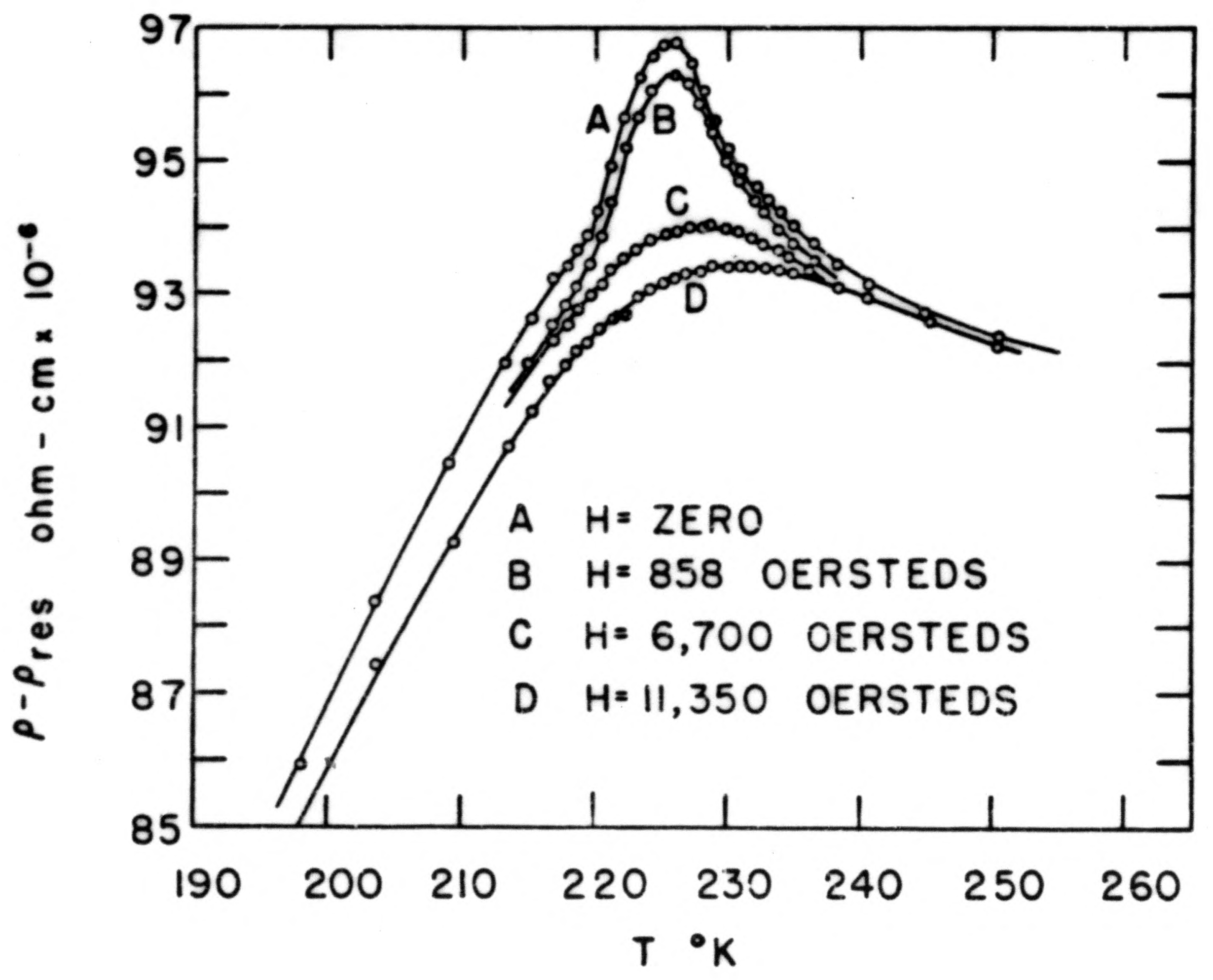

Fig. 12. Electrical resistivity (residual subtracted) for the c-axis crystal with a magnetic field applied along a b-axis. 
for the tripositive free ion. Thoburn et al. ${ }^{1}$ report a saturation moment of 9.25 Bohr magnetons per atom for polycrystalline terbium. Liu ${ }^{1}$ has suggested that the excess over the theoretical prediction may be due to the polarization of conduction electrons.

The effective moment in the paramagnetic region, obtained from the slope of the $\frac{1}{\mathrm{X}} \underline{\mathrm{vs}} \mathrm{T}$ plot, was $\mu_{\mathrm{eff}}=9.77$ Bohr magnetons for all three crystalline directions. The theoretical prediction is $\mu_{\text {eff }}=$ $\mathbf{g}[\mathrm{J}(\mathrm{J}+1)]^{\frac{1}{2}}=9.72$ Bohr magnetons, while Thoburn et $\underline{\text { al. }}{ }^{1}$ report $\mu_{\text {eff }}=9.7$ and Arajs and Colvin ${ }^{3}$ report $\mu_{\text {eff }}=9.62$.

The basal plane resistivity of tertium was found to be isotropic, within experimental error, over the temperature range covered in this investigation. The basal plane to c-axis anisotropy, given by $\frac{\rho_{a}}{\rho_{c}}$, was 1.29 at room temperature.

The room temperature resistivities of the terbium crystals were from 30 to $120 \%$ greater than the corresponding values for erbium and holmium reported by Green et al. ${ }^{13}$ and Strandburg et al. ${ }^{10}$, respectively. The room temperature anisotropy, however, is smaller than the value 1.7 reported for erbium and holmium by the same authors.

The sharp changes in slope in the resistivaty observed at $220 \pm 1^{\circ} \mathrm{K}$ for all three crystalline directions, which presumably occur at the ferromagnetic Curie temperature, agree very well with one of the determinations from the magnetic data, but not with the other. The weakly bound antiferromagnetic state results in considerable difficulty in determining the ferromagnetic Curie temperature accurately from the magnetic data, hence the change in slope of the resistivity is probably just as reliable an indication of the transition temperature. 
Mackintosh ${ }^{14}$ has proposed that extra planes of energy discontinuity are introduced into the Brillouin zone structurs as a result of the helical spin structure present in the antiferromagnetic phase of several of the rare earths. This causes a large change in the component of the Fermi surface vector along the c-axis while leaving the component in the basal plane relatively unchanged. He has suggested that this is the cause of the peak in the c-axis resistivity curve and postulated that if the antiferromagnetism could be quenched by the application of a magnetic field the peak would disappear.

The antiferromagnetic ordering is very easily overpowered in terbium, and he c-axis resistivity in a magnetic field behaved as Mackintosh predicted, as can be seen in Fig. 12. The slight decrease in resistivity below $220^{\circ} \mathrm{K}$ with the field on is attributed to a slight increase in the magnetic order which causes a decrease in the magnon scattering term.

\section{ACKNOWLEDGMENTS}

The authors wish to express their appreciation to J. E. Powell for the preparation of the rare-earth salts, to A. H. Daane and C. Habermann for the preparation of the metals, and to H. E. Nigh for cooperation in the preparation of the single crystals. 


\section{REFERENCES}

*Contribution No. 1220. Work was performed in the Ames Laboratory of the U. S. Atomic Energy Commission.

${ }^{1}$ W. C. Thoburn, S. Legvold, and F. H. Spedding, Phys. Rev. 112, 56 (1958).

${ }^{2}$ H. Leipfinger, Z. anorg. u. allgem. Chem. 231, 138 (1937).

${ }^{3}$ S. Arajs and R. V. Colvin, J. Appl. Phys. 32, 336S-337S (1961).

${ }^{4}$ L. D. Jennings, R. M. Stanton, and F. H. Spedding, J. Chem. Phys. 27, 909 (1957).

${ }^{5}$ w. C. Koehler, private communication.

${ }^{6}$ R. V. Colvin, S. Legvold, and F. H. Spedding, Phys. Rev. 120, 741 (1960).

${ }^{7}$ F. H. Spedding and J. E. Powell, J. Metals 6, 1131 (1954).

${ }^{8}$ F. H. Spedding and A. H. Daane, J. Metals 6, 504 (1954).

${ }^{9}$ P. M. Hall, S. Legvold, and F. H. Spedding, Phys. Rev. 117, 971 (1960).

${ }^{10}$ D. L. Strandburg, S. Legvold, and F. H. Spedding, Phys. Rev. (to be published).

11 J. K. Alstad, R. V. Colvin, and S. Legvold, Phys. Rev. 123, 418 (1961).

${ }^{12}$ S. H. Liu, Phys. Rev. 123, 470 (1961).

${ }^{13}$ R. W. Green, S. Legvold, and F. H. Spedding, Phys. Rev. 122, 827 (1961).

${ }^{14}$ A. R. Mackintosh, Phys. Rev. Letters 9, 90 (1962). 


\section{FIGURE CAPTIONS}

Fig. 1. Magnetic moment/g vs field for the a-axis crystal.

Fig. 2. Magnetic moment/g vs temperature for the a-axis crystal.

Fig. 3. Magnetic moment/g vs temperature for the a-axis crystal in the neighborhood of the Néel point.

Fig. 4. The reciprocal of the susceptibility $\left(\frac{1}{X}\right)$ vs temperature for the a-, b-, and c-axis crystals.

Fig. 5. Magnetic moment/g vs field for the b-axis crystal.

Fig. 6. Magnetic moment/g vs temperature for the b-axis crystal.

Fig. 7. Magnetic moment/g vs temperature for the b-axis crystal in the neighborhood of the Néel point.

Fig. 8. Magnetic moment/g vs $1 / \mathrm{H}$ for the b-axis crystal.

Fig. 9. Saturation magnetization of the b-axis crystal vs $T^{5 / 2}$.

Fig.10. Magnetic moment/g vs field for the c-axis crystal.

Fig.11. Electrical resistivity (residual subtracted) vs temperature for the a- and c-axis crystals and for a polycrystalline sample.

Fig.12. Electrical resistivity (residual subtracted) for the c-axis crystal with a magnetic field appiied along a b-axis.

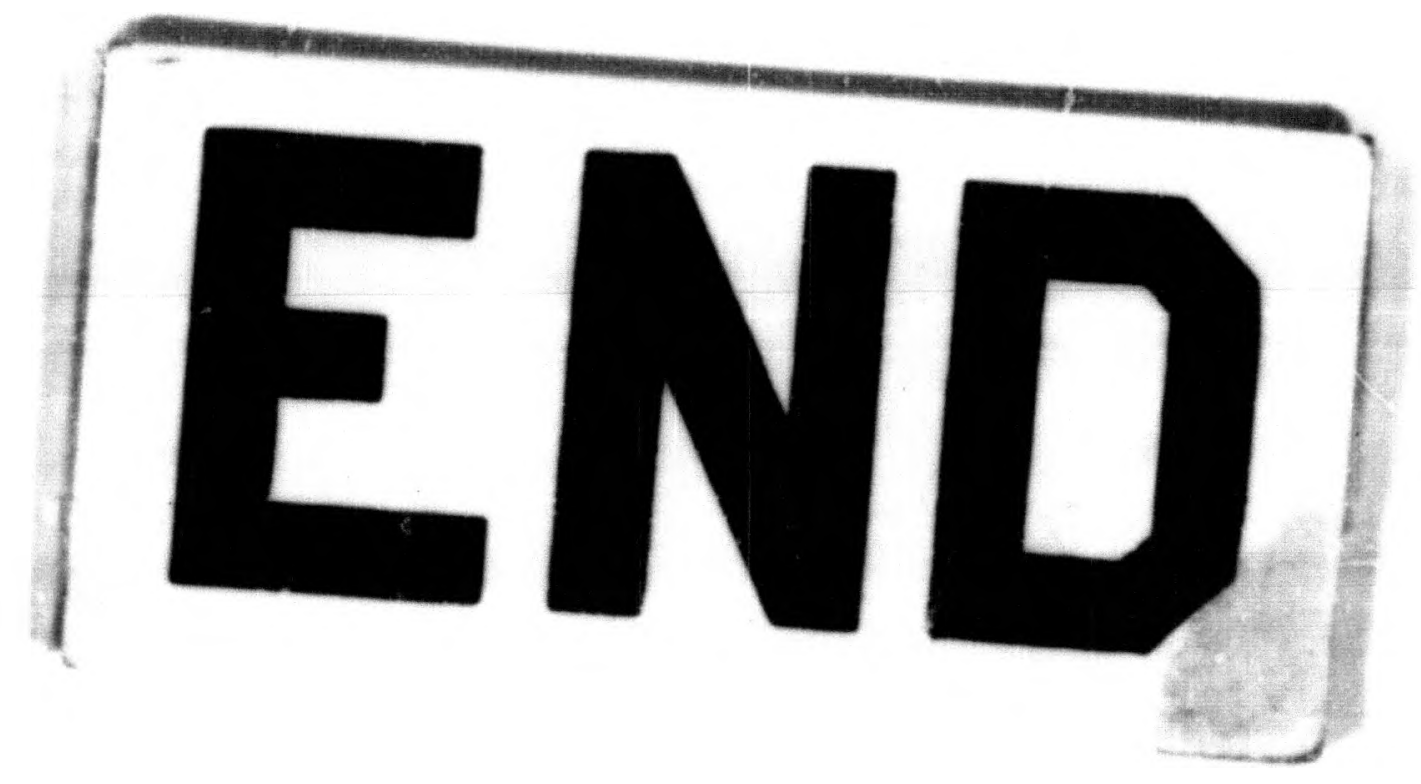

